\title{
The "value" of values-driven data in identifying Indigenous health and climate change priorities
}

\author{
Jamie Donatuto ${ }^{1} \cdot$ Larry Campbell $^{1} \cdot$ William Trousdale $^{2}$
}

Received: 24 May 2019 / Accepted: 31 October 2019 / Published online: 30 November 2019

(C) The Author(s) 2019

\begin{abstract}
Scholars worldwide have stated that some of the most devastating health impacts from climate change are experienced by Indigenous peoples. Yet, climate change health assessments rely primarily on technical data from climate models, with limited local knowledge and little to no values-driven community data. Values-driven data provide important information about how people define what health means, their health priorities, and preferred actions to maintain or improve health. The Swinomish Indian Tribal Community (Washington State, USA) developed and implemented a climate change health assessment founded on values-driven data. Successfully eliciting, structuring, and ultimately summarizing community values were achieved by incorporating tools and techniques from western disciplines (e.g., decision science, behavioral research) with local indigenous ways of learning and sharing. Results demonstrate that the assessment meaningfully engaged community members and effectively added their knowledge and values into the decision process. The assessment results protect and strengthen Swinomish community health and well-being by elevating health priorities, focusing limited energy and resources, ensuring that community members and others are working toward common goals, and establishing agreement around intended outcomes/results.
\end{abstract}

Keywords Community health $\cdot$ Indigenous $\cdot$ health impacts $\cdot$ Health assessment $\cdot$ Values

\section{Introduction}

"Our health comes from our culture and our culture comes from our lands, our waters. To make good decisions, these connections must be acknowledged."-Swinomish Elder.

Jamie Donatuto

jdonatuto@swinomish.nsn.us

1 Swinomish Indian Tribal Community, 17337 Reservation Rd, La Conner, WA 98257, USA

2 EcoPlan International, 208-131 Water Street, Vancouver, British Columbia V6B 4M3, Canada 
The effects of climate change on health are multiple, wide-ranging, and significant globally, nationally, and in the northwest USA (Smith et al. 2014; USGCRP 2018). Some of the largestscale and most devastating impacts from climate change are to the health and wellbeing of Indigenous peoples (Nakashima et al. 2018; Norton-Smith et al. 2016). In many parts of the USA, Tribes ${ }^{1}$ are leading the effort in assessing climate change impacts and planning adaptation actions. One of many reasons behind this push by Tribes is that reservation boundaries are not mobile, so Tribes must address the changes taking place in their homelands (Norton-Smith et al. 2016).

It is widely acknowledged that successful responses to challenges such as climate change should use a collaborative, co-learning approach, one that is guided by the values and priorities of those being impacted, as well as informed by the best available science (Dubois et al. 2018; Morgan and Di Giulio 2018). This requires engagement of multiple partners within and outside the community to improve the quality and legitimacy of decisions while enhancing capacity, trust, and understanding (Dietz 2013; NRC(National Research Council) 2008; USEPA 2009). Engagement done well will result in better climate adaptation decision-making; engagement done poorly may actually make matters worse (Ford et al. 2016; NRC 2008).

For community members to meaningfully engage, they require information about the consequences of climate change on things that they care about, and the opportunity to deliberate and provide input on the importance of the identified impacts. Then, the outcomes of deliberation can be integrated into decision-making (Capstick et al. 2016; Chilvers et al. 2014; Pidgeon and Fischhoff 2011). While significant progress is being made on promoting deliberative processes in general (e.g., International Association of Public Participation), there are few methods available to measure and assess social and cultural impacts as part of a deliberative process (Arvai and Post 2012; Donatuto et al. 2011; Satterfield et al. 2013). The term "social and cultural impacts" refers to effects that impair an individual or community's deeply held beliefs and values (worldviews), as well as activities associated with those beliefs and values. These beliefs and values are not universal norms, but are unique to different subpopulations. In the risk literature, social and cultural impacts are often considered "intangibles" because they are difficult to measure with conventional methods (Satterfield et al. 2013). Yet the elusiveness of these intangibles to assessment does not make them less important. For many Indigenous populations, who often hold unique worldviews, social and cultural values are central to health and to decision-making processes (Arquette et al. 2002; Gregory et al. 2016).

Researchers have further lamented the lack of practical examples of how to evaluate values and promote meaningful community deliberation in order to understand priorities and perspectives as a key part of the decision-making process (Brest and Krieger 2010). A particularly glaring gap is the lack of Indigenous community-based case studies in the literature (for exceptions, see: Carroll et al. 2018; Donatuto et al. 2011, 2016; Failing et al. 2013; Gregory and Trousdale 2009; Reid et al. 2014).

This paper provides a new approach that integrates applications of decision analysis and behavioral research in a community engagement context. We present a place-based, valuesdriven case study of climate change health impact assessment from an Indigenous perspective in five steps as an example of a successful values-based methodology. The community-based approach is founded on both technical information representing the latest advances from localized climate projection models, and Indigenous knowledge, values, and priorities from

\footnotetext{
${ }^{1}$ We use the term Tribes in this paper because we work for Indigenous communities in the USA, where the term "Tribe" is accepted and most frequently used. We recognize that this term is not the universal norm. When we are speaking more broadly to Indigenous issues, we use the term Indigenous.
} 
the community members who are impacted by climate change. The results provide decision makers and climate adaptation teams with the technical data and values-based evidence to design better solutions that are more responsive to community priorities, more likely to be implemented, and more likely to be supported by the community.

\subsection{2 Community health from an Indigenous perspective}

The Swinomish Indian Tribal Community (SITC) is federally recognized Indian Tribe organized under Section 16 of the Indian Reorganization Act and governed by the Swinomish Indian Senate (Senate), comprising 11 elected representatives. Today, there are nearly 1000 enrolled members. The Swinomish Reservation is located on the southeastern end of Fidalgo Island in Washington State, USA. The Reservation was established by Article 2 of the Treaty with the Dwamish, Suquamish, Etc., 12 Stat. 927 (1855). The Reservation includes approximately 10,800 acres of upland area and approximately 4500 acres of wet and filled tidelands; most of the Reservation is ringed by salt water (Fig. 1). Harvesting, preparing, and using first foods and resources (also called traditional foods, or "our foods" by community members) are an integral part of the social and cultural fabric in the Swinomish community. The Swinomish are fishing, hunting, and gathering people; generations since time immemorial have been and continue to be integrally connected to the land, water, air, and natural resources. Akin to many other Indigenous communities, Swinomish people characterize a healthy community by referencing countless generations of knowledge and practices developed via these connections. In other words, "health" is shaped by the many interrelated relationships between humans, non-human beings, nature, local natural resources, and the spiritual realm (i.e., social and cultural values). Moreover, health is thought of on familial and community scales, rather than an individual scale. And while biophysical health certainly plays a role in Swinomish views of health, it is not the sole or primary factor. In Swinomish beliefs, physical health status is often the outcome of health status in the social, cultural, mental, environmental, and intellectual realms (Donatuto et al. 2011, 2014, 2016).

Swinomish staff Campbell and Donatuto developed a set of health indictors derived from community input to reflect the nonphysical aspects (intangibles) of health important to the community. Figure 2 depicts the six Swinomish Indigenous Health Indicators (IHIs): cultural use, community connection, self-determination, resiliency, education (intergenerational knowledge transfer), and natural resource security (Donatuto et al. 2016). The IHIs present a positive vision of health, in lieu of a status of disease. The grouping of words for each term can be considered a distillation of meaning from one worldview to another - at top is the Lushootseed term, which often does not have a direct English translation, yet embodies the land, water, animals, stories, and people in a manner that English terms are unable to capture in definition. The Lushootseed term is summarized by the English words commonly used by community members at the base of each IHI group. The English words are then "translated" into phrases more easily understood by researchers and decision-makers outside of Swinomish, as seen in the middle phrase. The distillation of terms exemplifies the work involved in developing Swinomish-specific health values, and how Swinomish health priorities are not present in conventional health measures. The IHIs are meant to be employed in parallel with other health assessments already in use, such as technical data-based community health assessments. The purpose of the parallel assessment technique is to ensure that the information, and values, in the IHI are not subsumed piecemeal into an established framework, which cannot and will not value the unique indicators and the values they represent. 


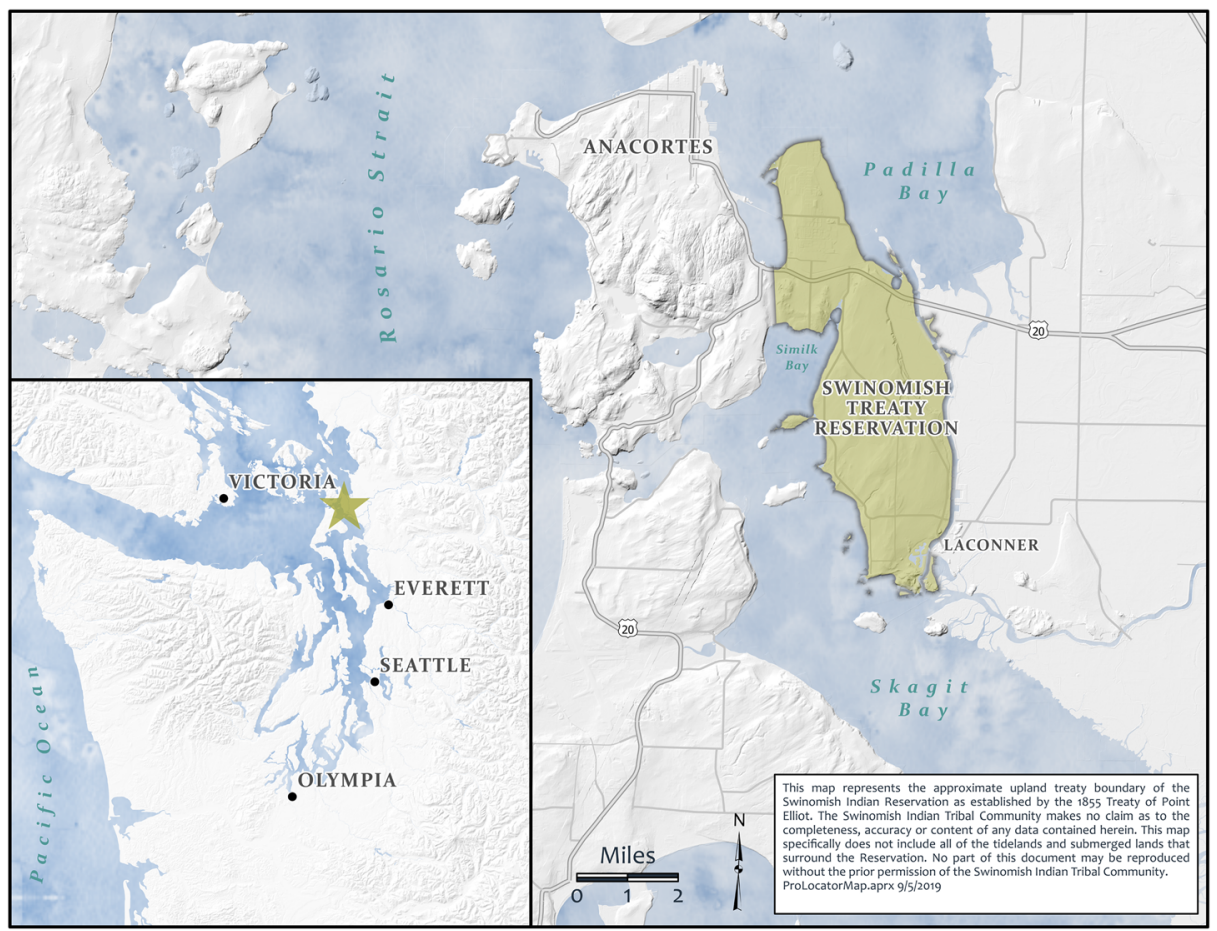

Fig. 1 Location of the Swinomish Indian Tribal Community in Washington State, USA

We began developing the IHI to assess community health impacts from contamination (Donatuto et al. 2011, 2016). We then tested the IHI for use in a climate change context with two Indigenous communities (Donatuto et al. 2014). With promising results, we expanded on the initial methods to develop a 5-step process for evaluating climate change impacts on Swinomish community health.

\section{The engagement process: 5 steps of an Indigenous community health assessment}

In this section, we describe the methods used to engage community members in dialogue about climate-driven health impacts and to evaluate the information learned in the discussions. In lieu of presenting methods and results in separate sections, we discuss the methods and results for each step consequentially, as the results from previous steps are used in the methods of the subsequent steps.

Our lead team consisted of Swinomish staff Larry Campbell (wanaseah) and Jamie Donatuto and consultant William Trousdale. The Swinomish Senate reviewed and approved the project before work began. Our community engagement goals were to achieve effective, informed, and meaningful participation, which we hoped would yield legitimate and transparent results. These goals needed to be achieved within the practical limits of time and funding constraints (constraints that are present with all grant-funded projects). 


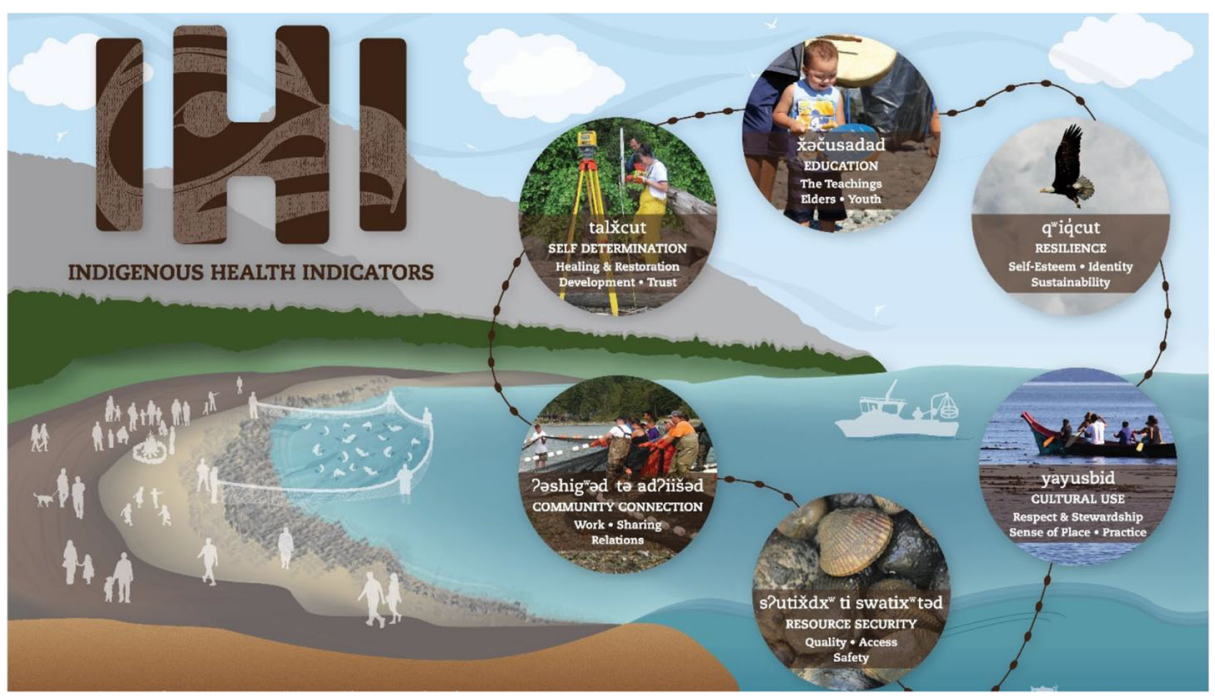

Fig. 2 Swinomish Indigenous Health Indicators. This infographic depicts a scene on the beach that demonstrates all six of the IHI in action. Families are working together beach seining (fishing with nets from the beach), steaming shellfish in a fire pit, and crab fishing in the bay. Elders are telling stories to younger generations; youth are exploring, and helping harvest, cook, and preserve the catch; the natural resources are accessible; people are asserting their sovereignty by being out on their lands and waters and engaged in culturally important practices, which "feed the body and the spirit" in the Swinomish way. The Figure was created by Emma Fox, Swinomish Communications Department

Our overall project hypothesis stated that when first foods are impaired due to climateinduced changes in habitat, the resulting amplification of negative effects reverberates throughout the social, cultural, mental, and physical health of Swinomish life. These negative effects can be evaluated using the Swinomish IHI as a framework and their relative importance determined through community-based value judgments and deliberation. This information would aid in site prioritization climate change interventions and support the creation of better adaptation actions. To test the hypothesis, we developed a five-step process that uses both technically driven data from computer modeling and values-driven data gathered via community engagement (Fig. 3).

From the onset of the project, Swinomish staff convened a community advisory board $(\mathrm{CAB})$, composed of community members, Elders, and staff. The CAB provided oversight, guidance, and advice throughout all five steps. The CAB imparted an additional and important review process of both the methods and the results, specifically focusing on ensuring that what was discussed expressed Swinomish values and priorities. At Swinomish, CABs are an essential and mandated part of all research projects. CABs provide direction as to what information can be shared, and which stories cannot be written down or included in project reports because the information is proprietary. They provide suggestions on how to avoid common pitfalls, such as information overload, jargon-filled questions, and confusing datasets. The $\mathrm{CAB}$ gave direction on participatory design, community dialogue, and preferred learning styles. For instance, while the term "first foods" is common in academia, it is not used within the Swinomish community. The CAB recommended referring to "Swinomish foods" or our foods instead of first foods in the workshops and interviews. The CAB also pilot-tested the methods described in this paper. Every part of the process detailed below was guided by insight from the $\mathrm{CAB}$. 


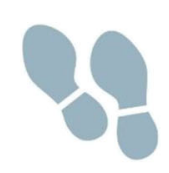

STEPS
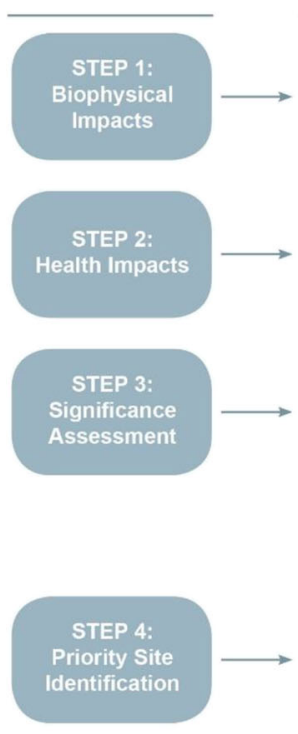

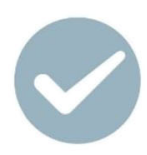

OUTCOMES

\section{QUESTIONS}

To what extent will

climate change affect 'Swinomish Food'?

What are the associated health impacts to the Tribe from changes to 'Swinomish Food'?

How important are these health impacts to the Tribe?

$\longrightarrow$
impact ranks at select sites (social, cultural, mental, and physical)

Biophysical impact crabs and clams)

\section{Value judgement ranks to determine $\mathrm{IHI}$ priorities and weights} ranks to critical habitat at select sites (salmon, Swinomish land is important, which are the priority sites where the Tribe should focus its effort and limited resources?
Ranking of priority sites (Direct Choice of community members)

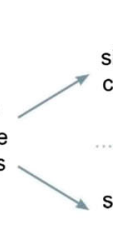

Ranking of priority sites (Value Weighted calculation using community value judgements)

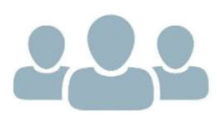

\section{WHO}

Swinomish Staff/ USGS scientists

Swinomish community members engaged in climate change work, CAB

\section{Swinomish community members, CAB}

\section{Swinomish} community members, $\mathrm{CAB}$

Swinomish Staff/ Consultants, CAB

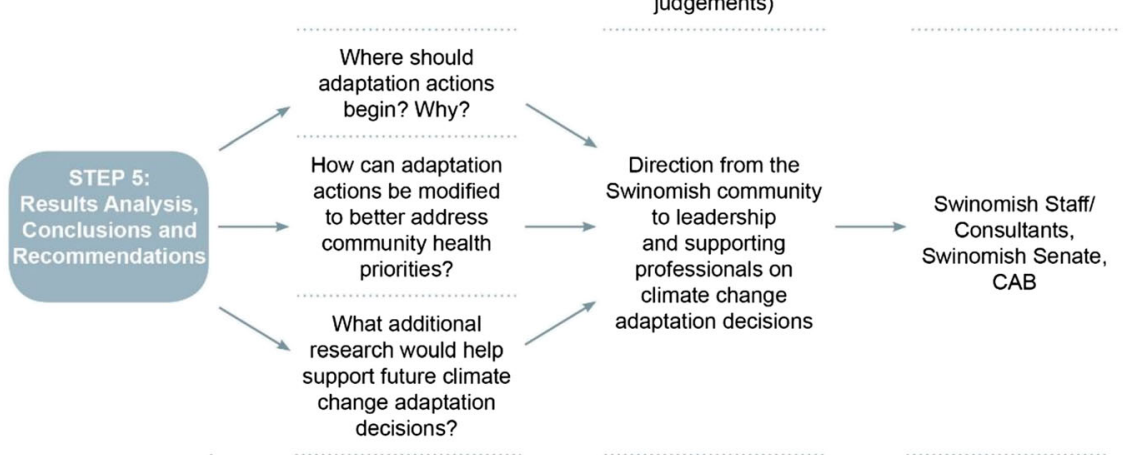

Fig. 3 Overview of Swinomish five step community health assessment process

\subsection{Step 1: forecasting biophysical climate impacts to priority natural resources}

Climate change impacts to aquatic habitats and species have the "potential for significant impacts on subsistence fishing Tribes" (USEPA 2014: 25). For this project, we chose to focus on salmon, clams, and crab, which all rely on Swinomish nearshore areas during one or more life phases and which are culturally important species for the Swinomish (Donatuto et al. 2011). These species represent a "tangible" proxy, providing context to community members' insights about climate change health impacts. Based on regional reports, staff anticipated that these species may be significantly impacted by projected climate changes. All five species of 
juvenile salmon out-migrate from the Skagit River; the delta of the Skagit is just south of the Reservation. Dungeness crab and multiple clam species are important resources found in the near shore habitats of the Reservation. Harvest of these natural resources is protected by Treaty right and highly valued by the Tribe.

As the infographic in Fig. 2 illustrates, nearshore areas are one of several important places for Swinomish people. The nearshore zone is highly vulnerable to both sea level rise and tidal surge inundation (SITC 2009). As a low-lying Reservation that is surrounded by water, developing actions to address sea level rise and tidal surge inundation risks are priorities for the Tribe, particularly when habitat for Swinomish foods and resources are at risk. For this step of the project, we worked with Swinomish fisheries staff and a climate modeling team from the US Geological Survey (USGS) (for more information on the model, see Barnard et al. 2014). We chose the six project sites based on current and historic use of the sites as harvest areas, a gradient of habitat types present, proximity to the Skagit River, exposure/effective fetch, and land uses (Fig. 4). The USGS team modeled projected impacts in the year 2100. Swinomish staff mapped data with high/low biological diversity and areas at greatest risk for beach loss due to sea level rise using updated elevation data and projections for the year 2100 . Figure 5 depicts the model's projection of flooding and the actual wrack line of debris (how high the tide surged) after a flood on the Swinomish Reservation. Note that the modeled projection accurately predicted the actual tidal inundation. The projected habitat losses forecasted in this step comprise the technical data used for the remainder of the assessment steps.

\subsubsection{Making technical data accessible and relevant to community members}

In order to present the model results to community members, the complex technical data analysis required distillation. Often, technical information is poorly understood by community members because scientists and engineers present results that overwhelm and confuse rather than simplify and clarify the information for those who need it to provide meaningful judgments. This can be a critical obstacle and leads to decisions being data driven rather than value driven (Trousdale and Gregory 2004). We sought to translate the data into a form that is meaningful to community members. This is not "dumbing down" the data; this is carefully curating the data to clearly present the modeling results, in this case with an emphasis on comprehensible visuals to communicate findings. Swinomish project staff and the $\mathrm{CAB}$ worked with scientists and a decision analyst to streamline the information. Feedback from the $\mathrm{CAB}$ included comments such as put less information in each image, make the information visually clear and easy to read with no small fonts, stay away from jargon such as "cobble" - say 'small rocks' instead, use a unit of measurement commonly used in the community (Fig. 6-no. 1 technical modeling).

Swinomish fisheries staff translated the technical information into simple decision-relevant performance measures (e.g., high risk, medium risk, low risk), then summarized the measures in a color-coded risk matrix (Fig. 6-no. 2 risk matrix). The risk matrix allowed for community members to quickly review risk levels to species at each site.

After Swinomish fisheries staff completed the risk matrix, we developed maps of each of the six sites using the information from the technical modeling and the risk matrix, contextualizing the data using photographic images of the beaches and overlaying projected impacts graphically (e.g., terms are over-laid in locations of each species' habitats). In the Lone Tree Point example in Fig. 6-no. 3, salmon habitat is at high risk, and crab and clam habitats are at medium-high risk. These graphics provide the information needed to understand projected changes while eschewing technical jargon and complex graphs and tables. 


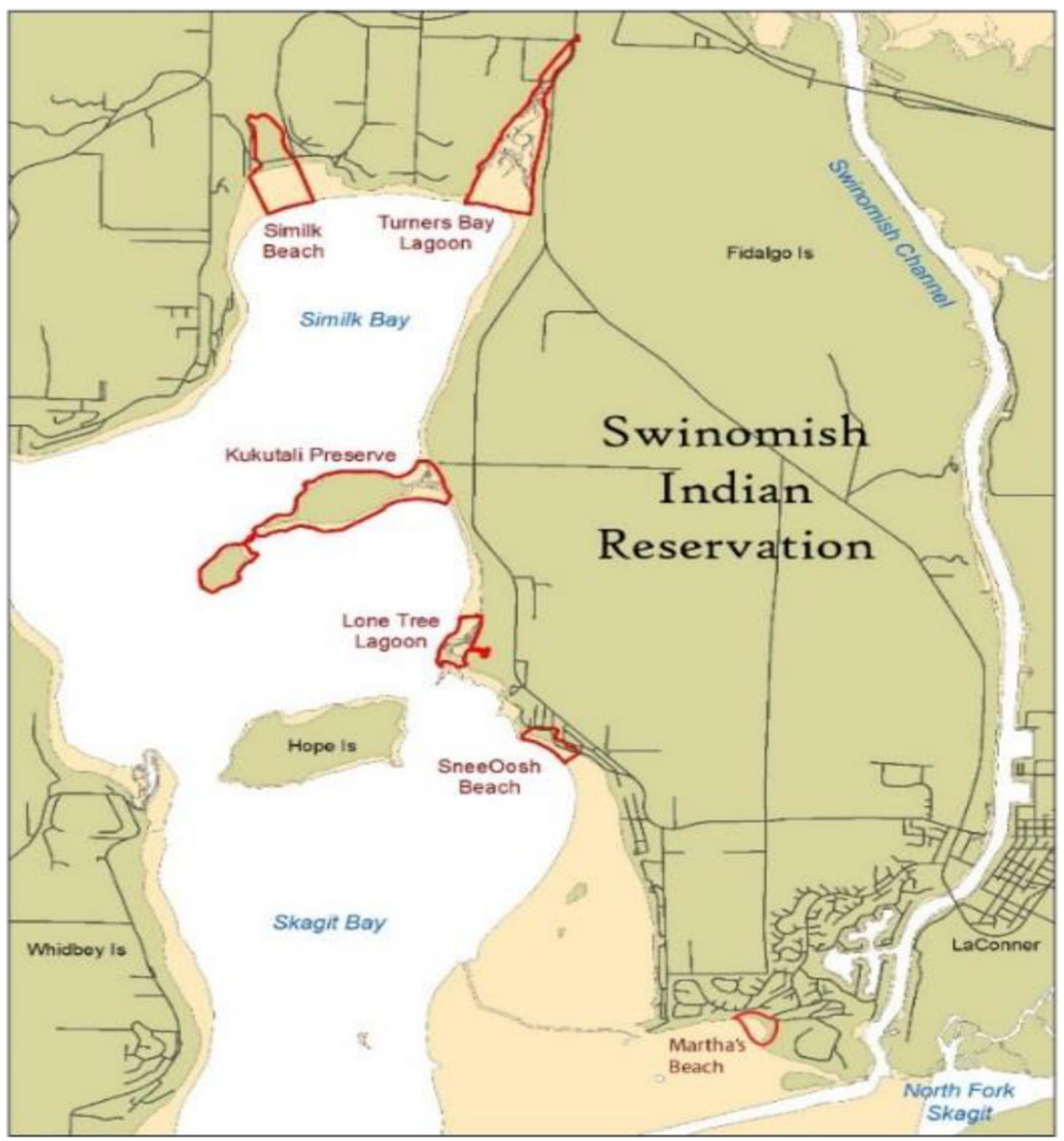

Fig. 4 Map of Swinomish Indian Reservation highlighting the six project focus areas (in red)

\subsection{Step 2: understanding community health impacts due to effects of climate change}

In Step 2, we used the results from the technical data analysis and modeling of potential impacts to first foods habitats described in Step 1 as the foundational knowledge to conduct an assessment of anticipated climate change health impacts at each site using the IHI as the community health evaluation framework. We implemented decision analysis procedures for systematically eliciting judgments of knowledgeable persons regarding the anticipated consequences. By facilitating a discussion, we were able to better understand the key uncertainties and data gaps, which then identified where to direct resources toward reducing those uncertainties and data gaps (Keeney and von Winterfeldt 1991).

We convened a workshop with a sub-set of Swinomish members who are well-versed in natural resources and climate impacts. The workshop included Elders and members of 


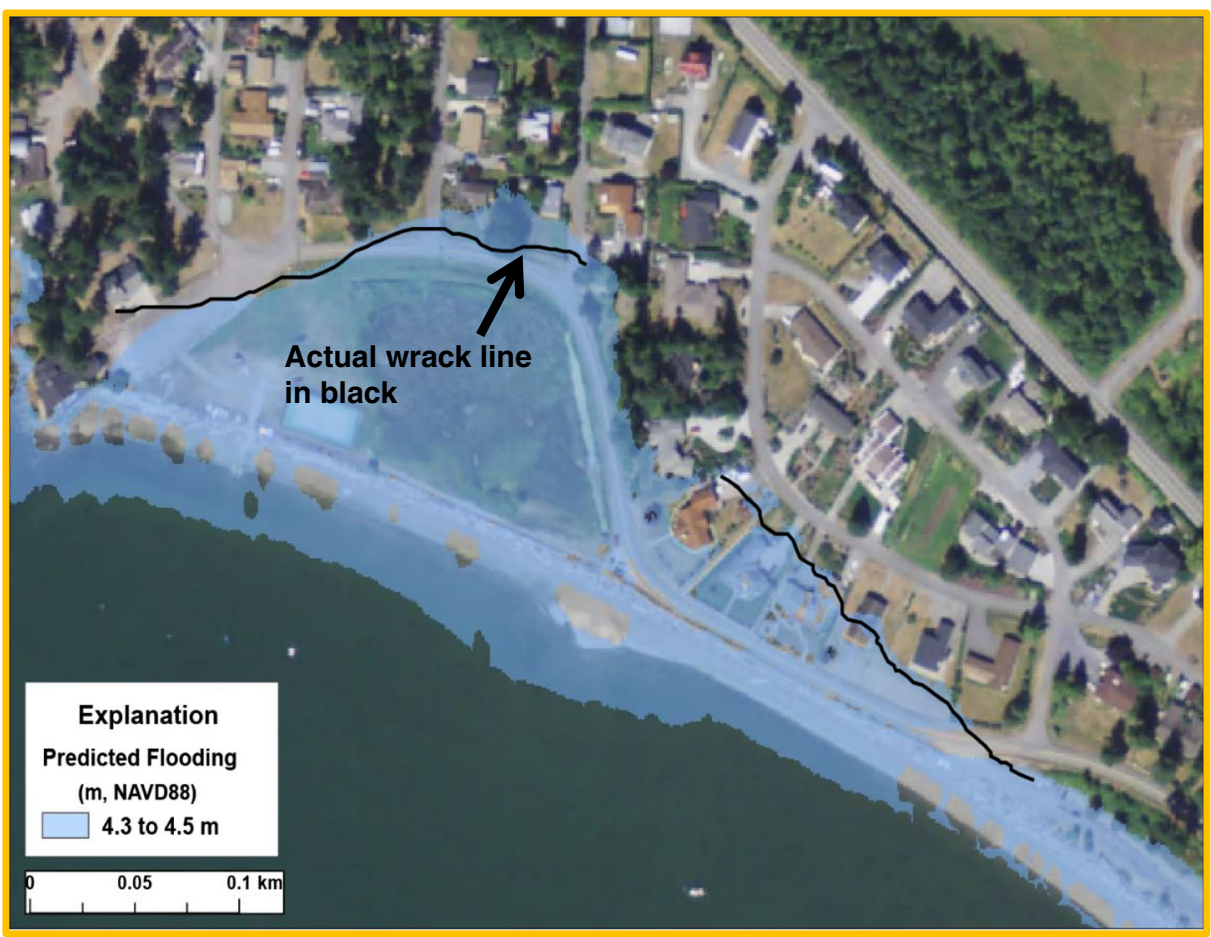

Fig. 5 Snee-Oosh area on Swinomish Reservation with measured wrack line (in black) after tidal inundation and predicted inundation (shaded blue) with the CosMoS model

the Swinomish Senate. We designed and implemented the workshop in two parts. The first part focused on understanding climate change risks to first foods and included a presentation of technical modeling of climate effects to Reservation nearshore sites and impacts to first foods at each of the six sites.

We conducted the second part of the process as three rounds of analysis, deliberation, and judgment elicitation on how impacts to first foods would affect IHI at each site. To promote independent thinking and reduce anchoring bias (e.g., from an influential or assertive member), we conducted the first round of evaluation by each participant using individual worksheets. A round of deliberation followed where participants discussed their scores and thinking behind them. A final round of evaluation asked each participant to reconsider and, if appropriate, modify or change their scores based on the deliberative exchange. As in the technical assessment, factors such as likelihood, extent, severity, duration, and recovery/adaptability were considered. In some cases, Swinomish staff conducted follow-up interviews to clarify scores.

The final result was an IHI criteria impact score for each site (Fig. 6-no. 4). For example, at Similk Beach, the impact to "community connection" was determined to be moderately low (equal to a numerical impact score of 2) while at Lone Tree, the impact would be "high" (equal to a numerical impact score of 5). These IHI impact scores would then be combined with the IHI value ranks elicited from community members in Step 3 to calculate a weighted priority rank discussed in Step 4. 

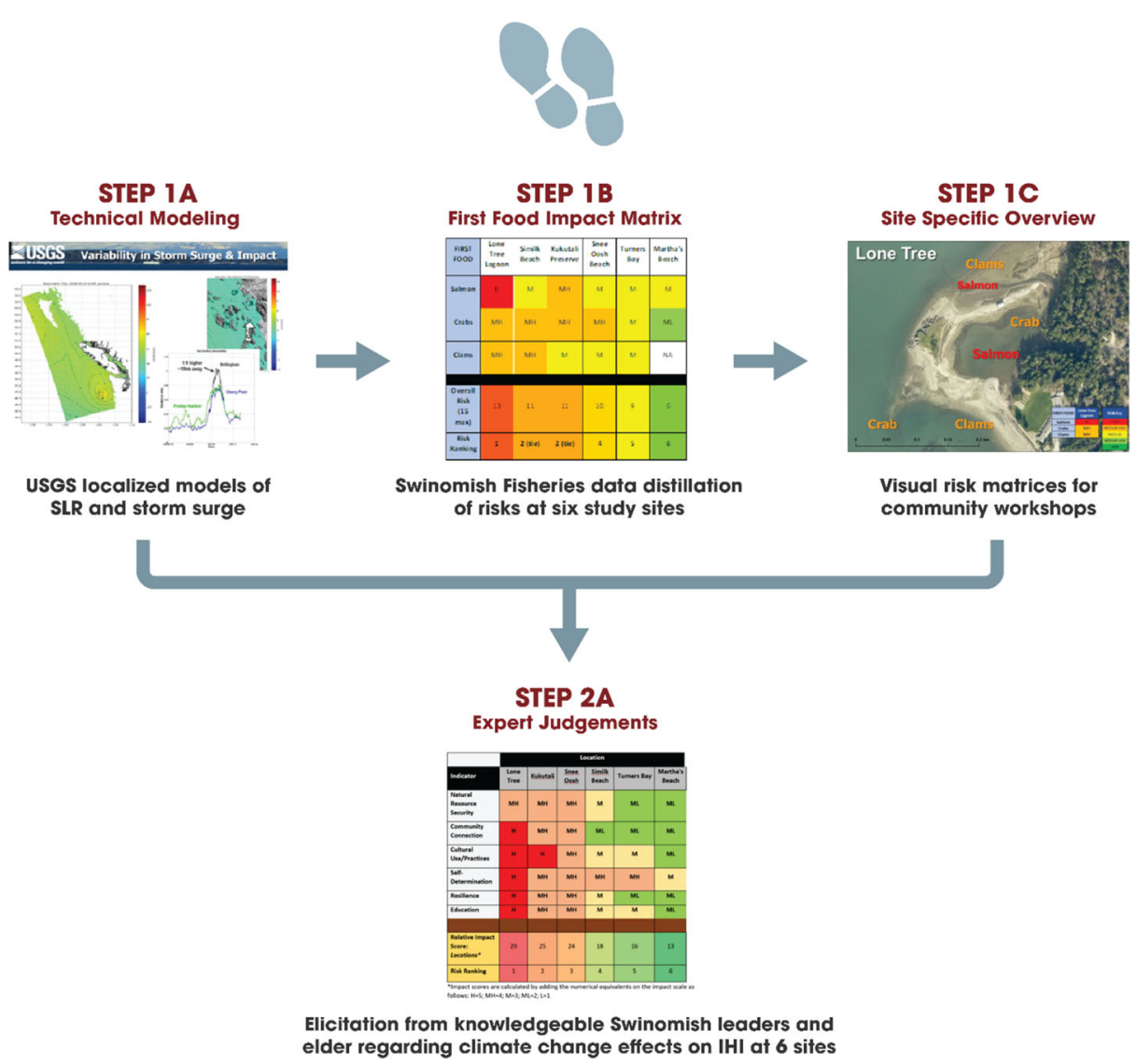

Fig. 6 Overview process of Steps 1 and 2: bringing technically driven data from computer modeling and valuesdriven data gathered via community engagement

\subsection{Step 3: determining the significance of health impacts based on community values}

Data collection in Steps 3 and 4 occurred together in the workshops; we have chosen to describe each step separately in order to be able to provide detail on the reasoning and analysis that occurred during each step.

While Steps 1 and 2 provided insights into climate change impacts at six specific sites, we wanted to know how important these impacts are relative to each other. So, Steps 3 and 4 involved engaging with a larger and more representative cross-section of community members in order to address the significance of the climate impacts to the community. The Swinomish community, akin to any other community, does not have a homogenous opinion set. We aimed for legitimacy by carefully identifying a diverse representation of community members and accountable decision makers as representatives of an array of community opinions. To achieve this, Swinomish staff worked with a range of demographic community groups (e.g., clam diggers, fishers, Elders, leadership, high school-aged youth) over the course of the engagement period (2016-2017). 
Knowing that time and resources limit staff from doing in-depth individual interviews with all community members, we chose to hold small facilitated group discussions in workshop settings. We went to the people, instead of expecting them to come to us; we organized workshops to take place during previously scheduled local events, such as Elders' lunch. We engaged high school students by visiting their classrooms. We hosted a bar-b-que on the beach when there was a clam dig so that we could speak with the clam harvesters. For interested individuals who were unable to attend a workshop, we organized individual interviews.

We provided food at all of the workshops. In many Indigenous communities, when you invite people to sit down at the table and share information, providing a meal is not a trivial part of the process; it may even be considered disrespectful to ask for an exchange of information without sharing food. Having in-depth knowledge of the community and its preferences of how to share knowledge, and with whom, are the keys to successful community data collection. Staff who facilitated workshops and held interviews are longtime employees of the Tribe, and some are Tribal members themselves. These familiar faces helped community members feel more comfortable in sharing their views and knowing that the information they shared would not be misused.

In total, we facilitated six workshops and numerous individual interviews. Sixty-eight Swinomish members deliberated over, provided judgments on, and indicated their priorities in response to climate change risks (about $14 \%$ of the local community). A total of $22 \%$ of participants identified as youth, $44 \%$ identified as adult, and $34 \%$ as Elders. We found a high level of agreement between the wide range of participants, which corresponds with research demonstrating that in participatory action research, smaller sample sizes can provide high-quality data (Rohrbach et al. 2016).

Measuring significance from the community perspective on the impacts to the IHI is essential if the goal is to identify not only priority sites, but also specify why they are a priority-key information when making decisions and designing adaptation responses. This value-based information can only come from the community. The workshops began with a poster session and one-on-one or small group discussions. Posters included the themes "Intro to climate change at Swinomish" and overview information from Step 1 and Step 2 (the risk matrices and images of the six sites indicating level of risks to crab, clams, and salmon). Participants moved around the room to examine posters and familiarize themselves with the project. Staffs were on hand to provide project details, answer any questions, and listen to stories and experiences relayed about the sites and first foods. The poster session allowed for thoughtful consideration of climate impacts and one-on-one discussions with Swinomish staff. When location allowed, Swinomish staff also provided participants with the same information in a PowerPoint presentation.

In the next stage of the workshop, participants placed color-coded "sticky dots" on posters to encourage reflection on relative significance of the anticipated impacts at each site and for each IHI. Each sticky dot color represented a ranking-e.g., very important, somewhat important, not important. This allowed the group to consider what others were thinking and encouraged discussion around priorities. Sticky notes could be added by participants if they wanted to share thoughts about the rankings or other aspects of the posters. This non-committal and quasi-anonymous (names are not on the stick dots, but everyone knew the other people in the room) "dotmocracy" activity was not used for final quantitative results, but to engage participants and begin a process of deeper understanding based on input from other participants (Fig. 7). 
The sticky dots on posters acted as a rehearsal for the final exercise. We provided each participant with a set of worksheets (similar to the posters shown in Fig. 7). Participants worked individually to complete a final round of prioritization by ranking the significance of climate change impacts to each IHI by answering the question: Knowing that all aspects of health (the IHI) are important, which are most important to protect from climate change? Participants numerically ranked the six IHI from " 1 " to " 6 ," with 1 being the first/ highest importance. The participants' responses on the worksheets comprise the data we used for the project analysis. Ties were allowed, meaning that participants could give more than one criteria a score of 1 indicating it was the most important to protect, but we asked them to think about and rationalize their scores in terms of the anticipated change from climate change - not the general importance of the criteria.

One of the key aspects of our engagement process was to encourage thoughtful and explicit assessments of individual priorities by giving participants the opportunity to revise their preferences as the evidence became better understood and new information was introduced through deliberation (c.f., Lichtenstein and Slovic 2006; Pidgeon et al. 2014). Aware of the many behavioral biases affecting thoughtful preference elicitation (e.g., framing bias, anchoring bias, confirmation bias, jumping to conclusions), we devised the workshops to incorporate several opportunities for learning and familiarization with the prioritization methods used, and to think more carefully with an open mind (Kahneman 2011). The informal but systematic workshop design helped participants develop better informed judgments, a critical step when participants arrive with little project information and have not considered yet why one site might deserve more attention than another (i.e., trade-offs) (Slovic 1995).

Identifying site and IHI priorities, and the reasoning behind them, required thoughtful consideration of trade-offs (e.g., one site might contain current productive shellfish beds while another site had the most productive clam beds in the past, but access has been forcibly denied

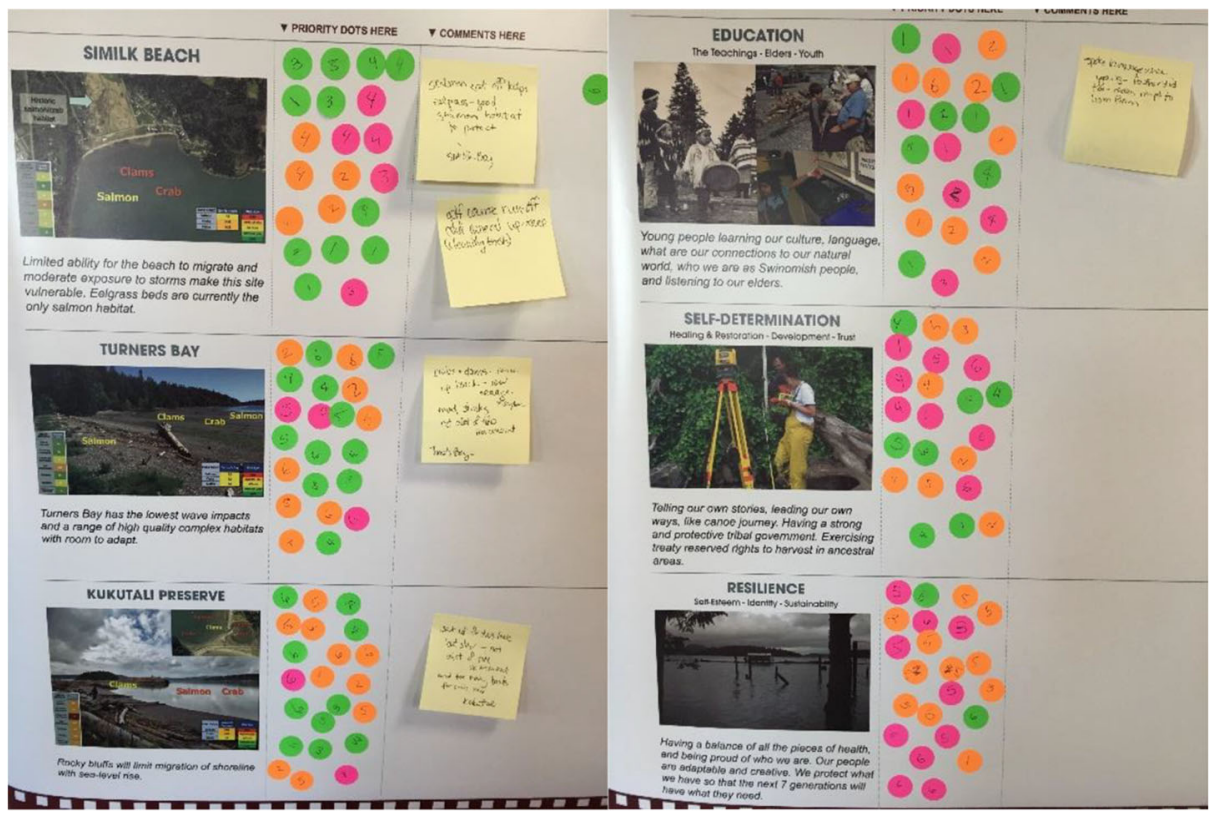

Fig. 7 Two examples of "dotmocracy" in action 
by non-native landowners). These choices can be difficult to make, especially across multiple sites and aspects of health, which may have differing degrees of importance between individuals or families. Furthermore, research has demonstrated that people's expressed preferences may fail to be captured in their eventually chosen priorities (Tversky et al. 1988) because key considerations are ignored (Bond et al. 2010), dominated by more easily measured, more tangible considerations (Gregory et al. 2012), or overpowered by metrics or considerations that are more commonly used in predominant assessment methodologies (Kovach 2010; Smith 2012). Our methodology is designed to actively counter these concerns.

The workshops started with climate change overview information and relatively simple tasks including straightforward questions that allowed participants to reflect on what is important to them (e.g., via dotmocracy, Fig. 7), before progressing to more difficult ones (worksheet exercises). This design fits with what we know about making things meaningful and measurable for people (e.g., measurement theory, see: Kahneman 2011; Krantz et al. 1971). The workshop approach stressed mutual learning so participants could think through judgment tasks from a common context. The value-based approaches used in the workshops have been shown to foster more informed judgments by framing the questions in multiple ways and asking for feedback on the initial iterations (Hobbs and Horn 1997). Lastly, to promote internal consistency, participants were asked weight the IHI criteria before ranking site alternatives (Bessette et al. 2014).

Swinomish staff anticipated that one of the challenges with engaging the community is that prioritizing sites and IHI can be uncomfortable. For many community members, all of the locations are important and all community health and wellness values are important: "don't make me choose - protect everything." We took care in the design process to come to an understanding that not all aspects of community health and wellness will be impacted by climate change to the same extent, and these impacts will differ from location to location. Further, given the Tribe's limited resources (money, time, capacity), not all protective actions could be undertaken immediately. Therefore, priorities are needed so that better decisions can be made. Characterizing the issues in this way promoted thoughtful discussion within the context of climate change using the evidence provided by multiple forms of scientific datawestern science and Indigenous.

The participants' IHI priority ranking judgments provided insightful results. As shown in Table 1, using average ranking as an indicator, the results indicate that education is the most important criteria to protect, with an average ranking score of 2.26. Furthermore, we found strong agreement among all workshops' participants that education is the most important indicator. This is important because it suggests that community members largely agree that actions protecting and promoting education will have more positive benefits to the health and wellbeing of Swinomish members and should garner a high level of support in climate adaptation planning. This information is provided to the Swinomish Senate in Step 5 in order to direct staff and adaptation experts to creatively modify or improve adaptation responses. Education was followed by natural resource security (2.56) and cultural use and practice (2.78).

Aggregation of participation data and using averages can be misleading as it does not provide insight into the range of agreement or disagreement. Failure to understand the extent and the size of value differences across individuals may be problematic. If the average is a result of high polarization within the community or if groups of individuals feel strongly and are ignored because they were lost in the averaging process, it may lead to false confidence and social conflict in decisions. Conversely, decision makers can act confidently if results show a 
high degree of agreement across participants. Swinomish therefore used the level of agreement as a way to validate results. Where we found weak agreement, it provided a signal that further engagement and analysis may be warranted. As indicated in Table 1, priority ranking for the top three IHI showed strong levels of agreement. Only the fifth-ranked self-determination revealed a weak level of agreement. Exploring age and demographic differences may help to tease out where and why levels of agreement are weaker. For example, for the fourth-ranked indicator, community connection, further analysis revealed that youth (less than 30 years) and Elders (more than 65 years) consistently ranked community connection much higher than the adult participants.

Ranking scores readily communicate priorities of which nearshore areas and IHI to focus attention on first. Additional valuable information is gathered through the deliberation and ultimate rationalization of those scores. For example, with the education ranking, one participant explained, "Traditional teachings from Elders explain our relationship to the land, how to connect with the world, and helps understand the world." Another stated, "Grandparents and parents taught us to take what we need - leave food for next season. Put food away important. We were never hungry. We were conservative providers. Only eat what you need, no more. We need access. Don't overuse it or it won't be there." And another, "Our Elders taught us how to heal ourselves with our foods, taught us to be proud of who we are. We are proud people and want generations coming up to learn too." Cultural use and practice relies on these sites, and as one Elder reminded us, "Carrying on the knowledge and celebrating very important." A participant explained that "[Resource Security] is a priority because without any of the foods to harvest, it would affect health, economy and cultural practice." Relating this to climate change, resilience needs to be considered: "Having sustainable food has been a way of life for us. If we can learn to adapt with the climate, we can learn to understand it."

\subsection{Step 4: prioritizing sites for adaptation action planning}

We used two methods to clarify Swinomish community members' site priorities: (1) direct ranking and (2) criteria weighting. Using more than one method to gain insight into priorities is useful. First, if results line up (i.e., convergence of priority ranking is

Table 1 Results of community evaluation of IHI

\begin{tabular}{|c|c|c|c|c|c|c|}
\hline Item & Education & $\begin{array}{c}\text { Natural } \\
\text { Resources } \\
\text { Security }\end{array}$ & $\begin{array}{c}\text { Cultural } \\
\text { Use and } \\
\text { Practices }\end{array}$ & $\begin{array}{c}\text { Community } \\
\text { Connection }\end{array}$ & $\begin{array}{c}\text { Self- } \\
\text { determination }\end{array}$ & Resilience \\
\hline $\begin{array}{c}\text { Score (average } \\
\text { rank) }\end{array}$ & 2.26 & 2.56 & 2.78 & 3.04 & 3.5 & 4.32 \\
\hline Priority Rank & 1 & 2 & 3 & 4 & 5 & 6 \\
\hline $\begin{array}{c}\text { Level of } \\
\text { Agreement** }\end{array}$ & Strong & Strong & Strong & Moderate & Weak & Moderate \\
\hline
\end{tabular}

**Level of agreement calculations: strong $=$ If over $50 \%$ of the participants ranked the criteria either 1-2 or 5-6 AND the difference between those that ranked the indicator 1-2 and those that ranked it 5-6 is less than 33\%. Moderate $=$ If over $50 \%$ of the participants ranked the criteria either 3.4 OR the difference between those that ranked the indicator 1-2 divided by those that ranked it 5-6 is between $33 \%$ and $66 \%$. Weak $=$ If over $50 \%$ of the participants ranked the criteria either 1-2 or 5-6 AND the difference between those that ranked the indicator 1-2 and those that ranked it 5-6 is greater than $66 \%$ 
found at either the aggregated or individual level), the priorities are validated. Second, where results are inconsistent between the two methods, the information can be used to provide focus areas where further research is needed: perhaps uncovering new information, key uncertainties, or missing values. Third, it may simply highlight where community members agree, disagree, or have misunderstandings. Insight into these issues allows staff to have productive and targeted follow-up engagement support. At the individual level, results of different methods are often inconsistent. When participants see the inconsistency themselves, it creates a space for them to think a little deeper about their priorities; our experience has been that it provides an opportunity for reflection and more open-minded group dialogue.

The first method was direct ranking by participants during the workshop sessions. The approach to ranking the sites followed the same process as the prioritization of IHI described in Step 3 with participants answering the question: Knowing that all Swinomish land is important, what are the priority locations (of the 6 sites) to focus the Tribe's limited resources and time on first? Again, participants placed sticky dots on posters as a first round of consideration, followed by ranking sites and IHI on individual worksheets. The combination of small group deliberation and in-person conversations between community members allowed local knowledge and stories that have been passed down by families to be shared. ${ }^{2}$

The second method, deriving value weighted ranks for site priorities, required that we convert community IHI priority rankings collected in Step 3 to normalized weights (Danielson and Ekenberg 2017; Roszkowska 2013). By converting the ranked priorities to weight, we created what is essentially a multi-criteria value index that can be used for calculating priorities using basic math (see methods in Keeney 1992). IHI weights for each participant were generated by the project team using the participant's ranking data and converting it into weights using the rank-ordered centroid (ROS) weighting method. ${ }^{3}$ These individual weights were then applied to the site-specific impact scores, providing a value-weighted prioritization. ${ }^{4}$

\footnotetext{
${ }^{2}$ We choose methods that did not record stories and other proprietary data. While it is important, and part of the educative process, for stories to be shared within the workshops, recording the stories puts them at risk to be shared outside of the community without free, prior, and informed consent. The quotes used in this paper are from the notes de-identified individuals wrote on their ranking worksheets. All results and dissemination, including this paper, have been reviewed and approved in accordance with Swinomish IRB procedures. See Harding et al. (2012) and Williams and Hardison (2013) for a more in-depth discussion of the myriad intellectual property risks that Indigenous communities face and how to avoid them.

${ }^{3}$ Several methods for selecting approximate weights were evaluated for this analysis, including rank sum (RS) and rank-order centroid (ROC) weights. The literature suggests that ROC weights may perform better than the other options in terms of choice accuracy (c.f., Roszkowska 2013).

${ }^{4}$ The relative IHI ranking judgments depend only on the marginal change from climate impacts to the IHI criteria. If the IHI criteria are considered fundamental by the participants, then independence is a reasonable assumption and an additive structure is appropriate. An additive function requires only basic math (multiplication and addition) to calculate overall value-weighted score. An additive function, given attributes $\mathrm{x} 1, \ldots, \mathrm{xN}$, can be written as follows:

$N$

$V\left(x_{1 . . .}, x_{N}\right)=\sum k_{i} u_{i}\left(x_{i}\right)$

$i=1$

where $V$ is the overall value (or utility) and the $k_{i}$ are the weights (or scaling constants) showing the relative contribution to the overall value from a change in a specific fundamental value, $x_{i}$. The $u_{i}$ are the single-attribute utility functions, one for each of the $x_{i}$ values. In this way, it is possible to establish clear mathematical relationships among the established values.
} 
Results of both methods are shown in Table 2 .

On average, the results of the community weighted prioritization and the direct ranking show that Lone Tree Point is the highest priority location to take action against climate change impacts - indicated by the convergence between the ranking methods and strong agreement between individuals' direct ranking results. Snee-Oosh Beach, Similk Beach, and Kukutali Preserve follow Lone Tree Point as the next priorities, but the order is not clear. Especially significant is the inconsistency between ranking methods at Kukutali Preserve, with the valueweighted method results rank it as the second most important site, but the direct ranking shows it as the fourth most important. Further, there is weak agreement between individuals on this direct ranking result. A review of comments implies that these results might be because of Kukutali access issues, resulting in fewer individuals knowing about and using the site. Those that do use the site currently, or have in the past, recognize its importance. These results are helpful in that they provide decision makers and the climate change adaptation planning team with a high degree of confidence in moving forward immediately on addressing potential impacts at Lone Tree Point, and that potential actions at the next level priority sites of SneeOosh Beach, Similk Beach, and Kukutali Preserve may be more informed.

As with the IHI, an improved understanding of community priority locations gained from the scoring is strengthened by articulating the group deliberation and rationale individuals provided for their ranking scores. For example, many individuals expressed deep connections to Lone Tree, such as this participant who stated: "Lone Tree is a special place for me, I spent many nights fishing and clamming here." Another explained the ranking of the priority sites this way: "Kukutali, Lone Tree, and Snee-Oosh are all ranked in the same way 'High'. This is due to having best / highest current community access and/or usage." Narrative responses are particularly useful in helping to understand priority rankings where there is a "weak" level of agreement, such as Kukutali Preserve. Here, one participant explained his high ranking: "Kukutali has historic importance" and another: "This site is significant to Elders who grew up here in the area and utilized as clam digging." Martha's Beach was ranked low by most participants. Participants provided comments such as, "it's [Martha's Beach] been dying for years."

\subsection{Step 5: results analysis and recommendations}

In the final step, 5, we collated the findings and presented the results to the Swinomish Senate, the governing body of the Tribe, for review and approval.

The purpose of this project was to meaningfully engage the Swinomish community in order to better understand community health impacts and priorities in the context of climate change. The findings confirmed the community health impact assessment hypothesis: adverse impacts to first foods will have widespread negative health effects on the Swinomish community. These health effects were successfully measured and evaluated using participatory qualitative and quantitative deliberative methods designed with the CAB. The results of this process provide decision makers and staff developing climate adaptation responses with clear community priorities as well as areas where community members do not have strong agreement.

The multiple methods approach we used verified that the community strongly agrees on the need to prioritize actions to address climate change impacts at Lone Tree Point, Snee-Oosh Beach, and Similk Beach. The process design also helped clarify the differences that arose from the diversity of participants' values, interests, and concerns. No community will have homogeneous views, Indigenous or otherwise. But well-designed processes can alert decision 
Table 2 Results of community prioritization of the six study sites: value-weighted and direct ranking methods

\begin{tabular}{|c|c|c|c|c|c|c|}
\hline & $\begin{array}{c}\text { Lone } \\
\text { Tree }\end{array}$ & $\begin{array}{c}\text { Snee- } \\
\text { Oosh } \\
\text { Beach }\end{array}$ & $\begin{array}{c}\text { Similk } \\
\text { Beach }\end{array}$ & $\begin{array}{c}\text { Kukutali } \\
\text { Preserve }\end{array}$ & $\begin{array}{c}\text { Martha's } \\
\text { Beach }\end{array}$ & $\begin{array}{c}\text { Turners } \\
\text { Bay }\end{array}$ \\
\hline $\begin{array}{c}\text { Weighted Ranking* } \\
\text { (average) }\end{array}$ & 1 & 3 & 4 & 2 & 6 & 5 \\
\hline Direct Ranking & 1 & 2 & 3 & 4 & 5 & 6 \\
\hline (average) & Strong & Strong & Strong & Weak & Weak & Moderate \\
\hline
\end{tabular}

*Average rank based on weighted IHI criteria Swinomish community meetings $\times$ impact score from technical modeling

**See below for agreement level definition. This was only applied to the direct ranking method. Level of agreement was calculated: strong = If over $50 \%$ of the participants direct ranked the criteria either 1-2 or 5-6 AND the difference between those that ranked the indicator 1-2 and those that ranked it 5-6 is less than 33\%. Moderate $=$ If over $50 \%$ of the participants direct ranked the criteria either 3-4 OR the difference between those that ranked the indicator 1-2 divided by those that ranked it 5-6 is between $33 \%$ and $66 \%$. Weak $=$ If over $50 \%$ of the participants direct ranked the criteria either 1-2 or 5-6 AND the difference between those that ranked the indicator 1-2 and those that ranked it 5-6 is greater than $66 \%$

makers to agreements and differences and indicate appropriate next steps. For example, the weak level of agreement regarding Kukutali Preserve is a useful measure to identify potential community conflict and protect values before action is taken.

The value-based, participatory approach provided additional insights that purely technically driven data research could not uncover. Without meaningful engagement of the community, the identified priorities would have been different. For example, the scientists' models indicated that the impacts to first foods at Snee-Oosh were ranked fourth out of the six sites, but the community identified Snee-Oosh as the second highest priority site.

We clarified an important distinction regarding identified impacts by using the five-step process. Evidence from technical-based impact information (e.g., modeling of what changes can most likely be expected) is different than value-based preferences elicited via community prioritization (e.g., how important are these changes). Technical experts have the knowledge and skills to provide information on the biophysical impacts, which directly impacts first foods. Yet these experts do not have the knowledge or skills to make projections on IHI impacts. Identifying what is important and who is an "expert" capable of providing impact information are important steps in establishing the necessary knowledge base - which comes from both technical experts (Step 1) and community members (Step 2) - to make informed judgments on impacts and priorities (Steps 3-5).

We were surprised that Education was ranked first with a high level of agreement across the community participants. In the beginning of the project, we presumed that natural resource security would be ranked first due to the context of using first foods in the assessment. While the results were not what we anticipated, they provide another example as to why impact assessments should not be considered an exercise for technical experts alone. The project produced clear evidence that rigorous community engagement provides insights that are unique and significant to informing climate adaptation decisions. With this project, we have provided a methodology for collection and integration of technical and value-driven data. 


\section{Conclusion}

This project effectively engaged the Swinomish community and successfully elicited knowledge and value judgments of community members. Collectively, these data can be used to support future efforts to protect and strengthen community health and well-being in response to climate change. The results will help to set priorities, focus limited energy and resources, ensure that community members and others are working toward common goals, and establish agreement around intended outcomes/results. As priority adaptation actions are identified and implemented over time, this information will support community leaders in both strategically allocating limited resources and justifying the selected actions.

Acknowledgments We gratefully acknowledge the Swinomish Indian Tribal Community and the project's Community Advisory Board for their leadership and guidance. We thank Dr. Robin Gregory and two anonymous reviewers for their thoughtful comments on the draft manuscript. Many thanks to Swinomish staff Sarah Grossman and USGS staff Dr. Eric Grossman for their technical data contributions.

Funding information Work funded by a US EPA grant to the Swinomish Tribe (no. RD-83559501); a CDC and National Indian Health Board grant to the Swinomish Tribe; and, a National Science Foundation grant to Decision Research (no. 1728807). Responsibility for the ideas expressed in this paper rest with the authors alone.

Open Access This article is distributed under the terms of the Creative Commons Attribution 4.0 International License (http://creativecommons.org/licenses/by/4.0/), which permits unrestricted use, distribution, and reproduction in any medium, provided you give appropriate credit to the original author(s) and the source, provide a link to the Creative Commons license, and indicate if changes were made.

\section{References}

Arquette M, Cole M, Cook K, LaFrance B, Peters M, Ransom J, Sargent E, Smoke V, Stairs A (2002) Holistic risk-based environmental decision making: a Native perspective. Environ Health Perspect 110(suppl 2):259264. https://doi.org/10.1289/ehp.02110s2259

Arvai J, Post K (2012) Risk management in a developing country context: improving decisions about point-ofuse water treatment among the rural poor in Africa. Risk Anal 32:67-80. https://doi.org/10.1111/j.15396924.2008

Barnard PL, van Ormondt M, Erikson LH, Eshleman J, Hapke C, Ruggiero P, Adams PN, Foxgrover AC (2014) Development of the Coastal Storm Modeling System (CoSMoS) for predicting the impact of storms on highenergy, active-margin coasts. Nat Hazards 74:1095. https://doi.org/10.1007/s11069-014-1236-y

Bessette DL, Arvai J, Campbell-Arvai V (2014) Decision support framework for developing regional energy strategies. Environ Sci Technol 48:1401-1408. https://doi.org/10.1021/es4036286

Bond SD, Carlson KA, Keeney RL (2010) Improving the generation of decision objectives. Decision. Analysis 7(3):238-255. https://doi.org/10.1287/deca.1100.0172

Brest P, Krieger LH (2010) Problem solving, decision making, and professional judgment: A guide for lawyers and policymakers. Oxford University Press, UK

Capstick SB, Pidgeon NF, Corner AJ, Spence EM, Pearson PN (2016) Public understanding in Great Britain of ocean acidification. Nat Clim Change 6(8):763. https://doi.org/10.1038/nclimate3005

Carroll C, Garroutte E, Noonan C, Buchwald D (2018) Using PhotoVoice to promote land conservation and Indigenous well-being in Oklahoma. EcoHealth 15(2):450-461. https://doi.org/10.1007/s10393-018-1330-9

Chilvers J, Lorenzoni I, Terry G, Buckley P, Pinnegar JK, Gelcich S (2014) Public engagement with marine climate change issues: (re)framings, understandings and responses. Global Environ Chang 29:165-179. https://doi.org/10.1016/j.gloenvcha.2014.09.006

Danielson M, Ekenberg L (2017) Tradeoffs for ordinal ranking methods in multi-criteria decisions. Group decision and negotiation: theory, empirical evidence and application. Selected Papers.

Dietz T (2013) Bringing values and deliberation to science communication. Proc Natl Acad Sci 110:1408114087. https://doi.org/10.1073/pnas. 1212740110 
Donatuto J, Satterfield T, Gregory R (2011) Poisoning the body to nourish the soul: prioritising health risks and impacts in a Native American community. Health Risk Soc 13:103-127. https://doi.org/10.1080 $/ 13698575.2011 .556186$

Donatuto J, Grossman EE, Konovsky J, Grossman S, Campbell LW (2014) Indigenous community health and climate change: integrating biophysical and social science indicators. Coast Manage 42(4):355-373. https://doi.org/10.1080/08920753.2014.923140

Donatuto J, Campbell L, Gregory R (2016) Developing responsive indicators of indigenous community health. Int J Environ Res Public Health 13(9):899. https://doi.org/10.3390/ijerph13090899

Dubois G, Stoverinck F, Amelung B (2018) Communicating climate information: traveling through the decisionmaking process. In: Serrao-Neumann S, Coudrain A, Coulter L (eds) Communicating Climate Change Information for Decision-Making. Springer Climate. Springer, Cham, pp 119-137. https://doi.org/10.1007 /978-3-319-74669-2 9

Gregory R, Trousdale W (2009) Compensating aboriginal cultural losses: an alternative approach to assessing environmental damages. J Environ Manage 90(8):2469-2479. https://doi.org/10.1016/j. jenvman.2008.12.019

Gregory R, Failing L, Harstone M, Long G, McDaniels T, Ohlson D (2012) Structured decision making: a practical guide to environmental management choices. John Wiley \& Sons

Gregory R, Easterling D, Kaechele N, Trousdale W (2016) Values-based measures of impacts to indigenous health. Risk Anal 36(8):1581-1588. https://doi.org/10.1111/risa.12533

Failing L, Gregory R, Higgins P (2013) Science, uncertainty, and values in ecological restoration: a case study in structured decision-making and adaptive management. Restor Ecol 21(4):422-430. https://doi.org/10.1111 j.1526-100X.2012.00919.x

Ford JD, Stephenson E, Cunsolo Willox A, Edge V, Farahbakhsh K, Furgal C, Harper S, Chatwood S, Mauro I, Pearce T, Austin S (2016) Community-based adaptation research in the Canadian Arctic. Wiley Interdiscip Rev Clim Change 7(2):175-191. https://doi.org/10.1002/wcc.376

Harding AK, Harper B, Stone D, O’Neill C, Berger P, Harris S, Donatuto J (2012) Conducting research with tribal communities: sovereignty, ethics, and data-sharing issues. Environ Health Perspect 120:6-10. https://doi.org/10.1289/ehp.1103904

Hobbs BF, Horn GT (1997) Building public confidence in energy planning: a multimethod MCDM approach to demand-side planning at BC gas. Energ policy 25(3):357-375. https://doi.org/10.1016/S0301-4215(97 )00025-6

Kahneman D (2011) Thinking, fast and slow. Farrar, Straus and Giroux, New York https://doi.org/10.1037 /h0099210

Keeney R (1992) Value focused thinking: a path to creative decision-making. Harvard University Press, Cambridge, Massachusetts

Keeney RL, Von Winterfeldt D (1991) Eliciting probabilities from experts in complex technical problems. IEEE T eng manage 38(3):191-201. https://doi.org/10.1109/17.83752

Kovach M (2010) Indigenous methodologies: characteristics, conversations and contexts. University of Toronto Press

Krantz DH, Luce RD, Suppes P, Tversky A (1971) Foundations of measurement, vol 1. Academic Press, New York

Lichtenstein S, Slovic P (eds) (2006) The construction of preference. Cambridge University Press

Morgan EA, Di Giulio GM (2018) Science and evidence-based climate change policy: collaborative approaches to improve the science-policy interface. In: Serrao-Neumann S, Coudrain A, Coulter L (eds) Communicating Climate Change Information for Decision-Making. Springer Climate. Springer, Cham, pp 13-28. https://doi.org/10.1007/978-3-319-74669-2_9

Nakashima D, Krupnik I, Rubis JT (2018) Indigenous knowledge for climate change assessment and adaptation. Cambridge University Press, Cambridge, U.K. and New York, NY. https://doi.org/10.1017 /9781316481066.002

NRC (National Research Council) (2008) Public participation in environmental assessment and decision making. National Academies Press, Washington, DC. https://doi.org/10.17226/12434

Norton-Smith K, Lynn K, Chief K, Cozzetto K, Donatuto J, Redsteer MH, Kruger LE, Maldonado J, Viles C, Whyte KP (2016) Climate change and indigenous peoples: a synthesis of current impacts and experiences. Technical Report PNW-GTR-944, United States Department of Agriculture, Forest Service, Pacific Northwest Research Station, Portland, OR.

Pidgeon N, Fischhoff B (2011) The role of social and decision sciences in communicating uncertain climate risks. Nat Clim Change 1:35-41. https://doi.org/10.1038/NCLIMATE108

Pidgeon N, Demski C, Butler C, Parkhill K, Spence A (2014) Creating a national citizen engagement process for energy policy. Proc Natl Acad Sci 111(Supplement 4):13606-13613. https://doi.org/10.1073 /pnas.1317512111 
Reid MG, Hamilton C, Reid SK, Trousdale W, Hill C, Turner N, Picard CR, Lamontagne C, Matthews HD (2014) Indigenous Climate Change Adaptation Planning Using a Values-Focused Approach: A Case Study with the Gitga'at Nation. J Ethnobiol 34(3):401-424. https://doi.org/10.2993/0278-0771-34.3.401

Roszkowska E (2013) Rank ordering criteria weighting methods - a comparative overview. Optimum Studia Ekonomiczne. 5(65):14-33

Rohrbach B, Anderson S, Laube P (2016) The effects of sample size on data quality in participatory mapping of past land use. Environment and Planning B: Planning and Design 43(4):681-697

Satterfield T, Gregory R, Klain S, Roberts M, Chan KM (2013) Culture, intangibles and metrics in environmental management. J Environ Manage 117:103-114. https://doi.org/10.1016/j.jenvman.2012.11.033

Smith KR, Woodward A, Campbell-Lendrum D, Chadee DD, Honda Y, Liu Q, Olwoch JM, Revich B, Sauerborn R (2014) Human Health: Impacts, adaptation, and co-benefits. In Field CB, Barros VR, Dokken DJ, Mach KJ, Mastrandrea MD, Bilir TE, Chatterjee M, Ebi KL, Estrada YO, Genova RC, Girma B, Kissel ES, Levy AN, MacCracken S, Mastrandrea PR, White LL (eds.) Climate Change 2014: Impacts, adaptation and vulnerability. part a: global and sectoral aspects. Contribution of Working Group II to the Fifth Assessment Report of the Intergovernmental Panel on Climate Change. Cambridge University Press, Cambridge, U.K. and New York, NY, pp 709-754.

Slovic P (1995) The construction of preference. Am psychol 50(5):364-371. https://doi.org/10.1037/0003-066 X.50.5.364

Smith LT (2012) Decolonizing methodologies: research and indigenous peoples. Zed Books, New York, NY

SITC (Swinomish Indian Tribal Community) (2009) Swinomish climate change initiative: impact assessment technical report. Office of Planning and Community Development, La Conner, WA. http://www.swinomishnsn.gov/climate_change/Docs/SITC_CC_ImpactAssessmentTechnicalReport_complete.pdf Last accessed May 24, 2019.

Trousdale W, Gregory R (2004) Property evaluation and biodiversity conservation Decision support for making hard choices. Ecol Econ 48:279-291. https://doi.org/10.1016/j.ecolecon.2003.09.011

Tversky A, Sattath S, Slovic P (1988) Contingent weighting in judgment and choice. Psychol rev 95(3):371-384

USEPA (2009) Valuing the protection of ecological systems and services A Report of the U.S. Environmental Protection Agency science advisory board. EPA-SAB-09-012

USEPA (2014) U.S. Environmental protection agency climate change adaptation plan. EPA 100-K-14-001

USGCRP (2018) Impacts, risks, and adaptation in the United States: Fourth National Climate Assessment, Volume II. Reidmiller DR, Avery CW, Easterling DR, Kunkel KE, Lewis KLM, Maycock TK, Stewart BC (eds). U.S. Global Change Research Program, Washington, DC, pp 1515. doi: 10.7930/NCA4.2018. https://nca2018.globalchange.gov/ Last accessed 18 March 2019.

Williams T, Hardison P (2013) Culture, law, risk and governance: contexts of traditional knowledge in climate change adaptation. Climatic Change 120:531-544. https://doi.org/10.1007/s10584-013-0850-0

Publisher's note Springer Nature remains neutral with regard to jurisdictional claims in published maps and institutional affiliations. 